\title{
Normal Algebraic Surfaces with Trivial Tricanonical Divisors
}

\author{
By
}

\author{
De-Qi Zhang*
}

\begin{abstract}
We prove that upto isomorphisms there are at least one and at most three rational log Enriques surfaces of index 3 and Type $A_{17}$.
\end{abstract}

\section{Introduction}

Let $T$ be a normal projective algebraic surface over the complex number field $\mathbf{C}$ with at worst quotient singular points (= Kawamata log terminal singular points in the sense of Kawamata and Kollár [Ka, Ko]). $T$ is called a $\log$ Enriques surface if the irregularity $\operatorname{dim} H^{1}\left(T, \mathcal{O}_{T}\right)=0$ and if a positive multiple $I K_{T}$ of the canonical Weil divisor $K_{T}$ is linearly equivalent to zero. Without loss of generality, we assume from now on that a log Enriques surface has no Du Val singular points (see the comments after [Z1, Proposition 1.3]).

The smallest $I$ such that $I K_{T} \sim 0$ is called the (global) index of $T$. It can be proved that $I \leq 66$ (cf. [Z1]). Recently, R. Blache [B1] has shown that $I \leq 21$. He also studied the "generalized" log Enriques surfaces where log canonical singular points are allowed.

Rational log Enriques surfaces $T$ can be regarded as degenerations of K3 or Enriques surfaces, which in turn played important roles in Enriques-Kodaira's classification theory for surfaces. Recently, V. A. Alexeev [A] has proved the boundedness of families of these $T$. In 3-dimensional case, the base surfaces

Communicated by Y. Miyaoka, October 16, 1996.

1991 Mathematics Subject Classification(s): Primary: 14J28,

Secondary: $14 \mathrm{~J} 26$

* Department of Mathematics, National University of Singapore, Lower Kent Ridge Road, Singapore 119260; E-mail: matzdq@nus.sg 
$W$ of elliptically fibred Calabi-Yau threefolds $\Phi_{|D|}: X \rightarrow W$ with $D \cdot c_{2}(X)=0$ are rational log Enriques surfaces [01-O5].

Let $T$ be a $\log$ Enriques surface of index $I$. The Galois $\mathbb{Z} / I \mathbb{Z}$-cover

$$
\pi: Y:=\mathscr{S P e c o}_{T} \oplus_{i=0}^{I-1} \mathcal{O}_{T}\left(-i K_{T}\right) \rightarrow T
$$

is called the canonical cover. Clearly, $Y$ is either an abelian surface or a $\mathrm{K} 3$ surface with at worst Du Val singular points. We note also that $\pi$ is unramified over the smooth part $T$-Sing $T$.

We say that $T$ is of Type $A_{m}$ or $D_{n}$ if $Y$ has a singular point of Dynkin type $A_{m}$ or $D_{n} ; \quad T$ is of actual Type $\left(\oplus A_{m}\right) \oplus\left(\oplus D_{n}\right) \oplus\left(\oplus E_{k}\right)$ if Sing $Y$ $=\left(\oplus A_{m}\right) \oplus\left(\oplus D_{n}\right) \oplus\left(\oplus E_{k}\right)$.

Around 1989, M. Reid and I. Naruki asked the author about the uniqueness of rational $\log$ Enriques surface of Type $D_{19}$. The determinations of all isomorphism classes of rational $\log$ Enriques surfaces of Type $A_{19}, D_{19}, A_{18}$ and $D_{18}$ have been done in [OZ1, OZ2].

In this series of three papers, we consider the cases $A_{17}$ and $D_{17}$. Actually, there is no rational $\log$ Enriques surface of Type $D_{17}$ (Theorem 4). In the Type $A_{17}$ case, the index $I$ is equal to one of $2,3,4,5,6,12$ by virtue of [Z3, Theorem 1; OZ5, the proof of Theorem 1]. Our main results are as follows:

Theorem 1. There is no rational log Enriques surface of Type $A_{17}$ and index $6 p$ for any positive integer $p$.

Remark 1. Consequently, a rational log Enriques surface of Type $A_{17}$ has index 2, 3, 4 or 5. The determinations of all isomorphism classes for the cases of index $I=2,4,5$, are done in $[\mathrm{Z3}, \mathrm{OZ} 5]$, while the case $I=3$ is treated in this note.

Theorem 2. Upto isomorphisms there is at least one and at most three rational log Enriques surfaces of index 3 and Type $A_{17}$. They are all of actual Type $A_{17}$ and isomorphic to one of $T_{i}(i=1,2,3)$ in Example 2.1.

Theorem 3. Let $T_{i}$ be as in Theorem $2, Y_{i} \rightarrow T_{i}$ the canonical cover and $g: X_{i} \rightarrow Y_{i}$ the minimal resolution. Write $\operatorname{Gal}\left(Y_{i} / T_{i}\right)=\left\langle\sigma_{i}\right\rangle$ where $\sigma_{i}$ is an automorphism of order 3 , and $\Gamma:=g^{-1}\left(\right.$ Sing $\left.Y_{i}\right)$ which is of Dynkin type $A_{17}$.

Then there are two smooth rational curves $F, H$ on $X_{i}$ such that $F+H+\Gamma$ is of Dynkin type $D_{19}$ and that the triplet $\left(X_{i},\left\langle\sigma_{i}\right\rangle, F+H+\Gamma\right)$ is isomorphic to Shioda-Inose's unique triplet $\left(S_{3},\left\langle g_{3}\right\rangle, \Delta_{3}\right)$ in [OZ1, Example 1] (see also 
Example 2.1 below), where $S_{3}$ is the unique K3 surface of Picard number 20 and discriminant 3.

Question 1. Determine whether $T_{i}$ and $T_{j}$ are not isomorphic to each other when $i \neq j$.

Remark 2. The answer to this question may not be easy if one looks at the long arguments in [OZ2, Theorem 1.6] in order to differentiate between two very symmetrically-constructed isomorphism classes of rational log Enriques surfaces of Type $A_{18}$.

Theorem 4. There is no rational log Enriques surface of Type $D_{17}$.

The organization of the paper is as follows. In $\S 1$, we consider automorphisms $\sigma$ of order 3 or 6 on K3 surfaces, and describe in detail the action of $\sigma$ around points lying on linear chains of smooth rational curves as well as the action of $\sigma$ on elliptic fibers. A precise relation between the numbers of $\sigma$-fixed isolated points and curves is obtained in Lemma 1.6 by applying the fixed point theorem for holomorphic bundles, which was proved by Atiyah, Segal and Singer in [AS1,2].

In $\S 2$, we construct precisely three rational $\log$ Enriques surfaces $T_{i}$ of index 3 and actual Type $A_{17}$. $\S 3$ and $\S 4$ are devoted to the proofs of the theorems.

\section{Acknowledgement}

I would like to thank Professor Y. Miyaoka for suggestions which improved the paper.

\section{$\S 1$. Preliminaries}

In this section, we shall fix the following notation:

$T$ is a rational $\log$ Enriques surface of index $I$ and $\pi: Y \rightarrow T$ is the canonical cover. $f: S \rightarrow T$ and $g: X \rightarrow Y$ are minimal resolutions. $\Sigma:=f^{-1}(\operatorname{Sing} T)$ and $\Gamma:=g^{-1}(\operatorname{Sing} Y)$ are reduced $f$-exceptional and $g$-exceptional divisors, respectively.

Note that $\pi$ is a Galois covering such that $\operatorname{Gal}(Y / T)=\mathbf{Z} / I \mathbf{Z}$ and 
$Y /(\mathbb{Z} / I \mathbb{Z})=T$. Clearly, there is a natural action of $\mathbb{Z} / I \mathbb{Z}$ on $X$ such that the minimal resolution $g: X \rightarrow Y$ is $(\mathbb{Z} / I \mathbb{Z})$-equivariant. We will need the following lemmas for the later use.

Lemma 1.1. Let $T$ be a rational log Enriques surface of index I with $Y$ the canonical cover. Then $\sigma^{*} \omega=\zeta_{I} \omega$ for exactly one generator $\sigma$ of $(\mathbb{Z} / \mathbb{Z})$, where $\zeta_{I}=\exp (2 \pi \sqrt{-1} / I)$ and $\omega$ is a non-zero holomorphic 2-form on Yor on X.

Proof. The result follows from the definition of $I$.

Lemma 1.2. With the notations and assumptions in Lemma 1.1, we have:

(1) The g-exceptional divisor $\Gamma$ is $\sigma$-stable.

(2) Every singular point on $Y$ has a non-trivial stabilizer subgroup of $\langle\sigma\rangle=\mathbb{Z} / I \mathbb{Z}$. In particular, every connected component of $\Gamma$ is $\sigma$-stable provided that I is prime.

(3) Every $\sigma^{i}$-fixed curve on $X$ where $\sigma^{i} \neq \mathrm{id}$, is contained in $\Gamma$ and hence a rational curve.

Proof. (1) is true because the singular locus $\operatorname{Sing} Y$ is $\sigma$-stable.

(2) follows from our additional assumption that $T=Y / \sigma$ has no Du Val singular points. (3) is true because $\pi: Y \rightarrow T$ is unramified outside the finite set Sing $T$.

Lemma 1.3. With the assumption and notation in Lemma 1.1, assume further that $I=p q$ for positive integers $p, q$. Then $Y_{1}:=Y /\left\langle\sigma^{q}\right\rangle$ is a rational $\log$ Enriques surface of index $p$ with the quotient morphism $Y \rightarrow Y_{1}$ as the canonical cover. Here we assume that $p>1, q>1$.

Proof. Ths follows from the fact that the (global) canonical index is equal to the 1.c.m. of local canonical indices.

The following result is proved in [OZ1, Lemmas 2.1 and 2.2].

Lemma 1.4. Let $X$ be a (smooth) $K 3$ surface with an order-three automorphism $\sigma$ such that $\sigma^{*} \omega=\zeta \omega$ for a non-zero holomorphic 2-form $\omega$ on $X$ and $a$ cubic root $\zeta$ of unity. Then the following statements are true.

(1) The fixed locus (point wise) $X^{\sigma}$ is a disjoint union of smooth curves 
and several isolated points.

(2) Suppose that $C_{1}+C_{2}+C_{3}$ is a linear chain of $\sigma$-stable smooth rational curves with $C_{2}$ as the middle component. Then exactly one of $C_{i}$ is $\sigma$-fixed.

(3) Let $C$ be a $\sigma$-stable but not $\sigma$-fixed smooth rational curve. Then there is a $\sigma$-fixed curve $D$ such that $C . D=1$.

Lemma 1.5 below is a consequence of Lemma 1.4 and Kodaira's classification of singular elliptic fibers. The condition $n \leq 18$ (resp. $n \leq 17$ ) in the type(2) (resp. the type(3)) comes from the fact that $\operatorname{rank} \operatorname{Pic} X<21$ (cf. [S, Cor. 1.5]).

Lemma 1.5. Let $X, \sigma$ be as in Lemma 1.4. Suppose that $\eta$ is a singular fiber of an elliptic fibration $\psi: X \rightarrow \mathbf{P}^{1}$ such that $\eta$ consists of $\sigma$-stable curves and contains at least one $\sigma$-fixed curve. (We note that every smooth rational curve on $X$ is $\sigma$-stable provided that $\sigma^{*} \mid \operatorname{Pic} X=\mathrm{id}$.) Then $\eta$ has one of the following types:

(1) $\eta=H_{1}+H_{2}+H_{3}$ is of Kodaira type $I V$, where $H_{i}$ 's share one and the same point. $H_{1}$ is the only $\sigma$-fixed curve in $\eta$.

(2) $\eta=H_{1}+H_{2}+\cdots+H_{n}$ is of Kodaira type $I_{n}$ with $H_{i} \cdot H_{i+1}=H_{n} \cdot H_{1}=1$ $(1 \leq i \leq n-1) . \quad n$ is either one of $3,6,9,12,15,18$. The curves $H_{1}, H_{4}, H_{7}$, $\cdots, H_{n-2}$ are the only $\sigma$-fixed curves in $\eta$.

(3) $\eta=H_{1}+H_{2}+2\left(H_{3}+H_{4}+\cdots+H_{n-2}\right)+H_{n-1}+H_{n}$ is of Kodaira type $I_{n-5}^{*}$ where $H_{1} \cdot H_{3}=H_{i} \cdot H_{i+1}=H_{n-2} \cdot H_{n}=1(2 \leq i \leq n-2) . \quad n$ is either one of $5,8,11,14,17 . H_{3}, H_{6}, H_{9}, \cdots, H_{n-2}$ are the only $\sigma$-fixed curves in $\eta$.

(4) $\eta=3 \mathrm{H}_{1}+2 \mathrm{H}_{2}+\mathrm{H}_{3}+2 \mathrm{H}_{4}+\mathrm{H}_{5}+2 \mathrm{H}_{6}+\mathrm{H}_{7}$ is of Kodaira type $\mathrm{IV}^{*}$ where $H_{1} \cdot H_{i}=H_{i} \cdot H_{i+1}=1(i=2,4,6) . \quad H_{1}$ is the only $\sigma$-fixed curve in $\eta$.

(5) $\eta=4 H_{1}+2 H_{2}+3 H_{3}+2 H_{4}+H_{5}+3 H_{6}+2 H_{7}+H_{8}$ is of Kodaira type $I I I^{*}$ where $H_{1} \cdot H_{i}=H_{j-1} \cdot H_{j}=H_{j} \cdot H_{j+1}=1(i=2,3,6 ; j=4,7) . \quad H_{1}, H_{5}, H_{8}$ are the only $\sigma$-fixed curves in $\eta$.

(6) $\eta=6 \mathrm{H}_{1}+3 \mathrm{H}_{2}+4 \mathrm{H}_{3}+2 \mathrm{H}_{4}+5 \mathrm{H}_{5}+4 \mathrm{H}_{6}+3 \mathrm{H}_{7}+2 \mathrm{H}_{8}+\mathrm{H}_{9}$ is of $\mathrm{Ko}$ daira type $I I^{*}$ where $H_{1} \cdot H_{i}=H_{3} \cdot H_{4}=H_{j} \cdot H_{j+1}=1 \quad(i=2,3,5 ; j=5,6,7$, 8). $H_{1}, H_{7}$ are the only $\sigma$-fixed curves in $\eta$.

Lemma 1.6. Let $X$ be a (smooth) K3 surface with an order-six automorphism $\sigma$ such that $\sigma^{*} \omega=\zeta \omega$ for a non-zero holomorphic 2-form $\omega$ on $X$ and a 6-th primitive roof $\zeta$ of unity. Let $P_{1}, P_{2}, \cdots, P_{M}$ (resp. $C_{1}, C_{2}$, $\cdots, C_{N}$ ) be all isolated points (resp. all irreducible curves) in $X^{\sigma}$.

Assume that each $C_{i}$ is rational. Then $C_{i}$ is smooth and disjoint from $C_{j}(i \neq j)$, 
and $M_{1}+M_{2} / 2=3(N+1)$ where $M_{1}, M_{2}$ are non-negative integers to be defined in the proof below satisfying $M_{1}+M_{2}=M$.

Proof. Since $\sigma^{*} \omega=\zeta \omega$, one has the diagonalization $\sigma^{*}=\operatorname{diag}\left(\zeta^{2}, \zeta^{-1}\right)$ or $\sigma^{*}=\operatorname{diag}\left(\zeta^{3}, \zeta^{-2}\right)$, with suitable local coordinates around $P_{i}$. Let $P_{i}$ for $1 \leq i \leq M_{1}$ (resp. $P_{j}$ for $M_{1}+1 \leq j \leq M_{1}+M_{2}=M$ ) be all isolated points in $X^{\sigma}$ such that $\sigma^{*}=\operatorname{diag}\left(\zeta^{2}, \zeta^{-1}\right)\left(\operatorname{resp} . \sigma^{*}=\operatorname{diag}\left(\zeta^{3}, \zeta^{-2}\right)\right)$ around $P_{i}\left(\operatorname{resp} . P_{j}\right)$.

Taking a point $P \in C_{k}$, we see that $\sigma^{*}=\operatorname{diag}(1, \zeta)$, with suitable local coordinates $(x, y)$ around $P$. Thus, around $P, X^{\sigma}$ is equal to $\{y=0\}$ and hence smooth. So $X^{\sigma}$ is a disjoint union of smooth curves $C_{k}$ 's and points $P_{l}$ 's.

We now calculate the holomorphic Lefschetz number $L(\sigma)$ in two ways as in [AS1, 2, pages 542 and 567]:

$$
\begin{gathered}
L(\sigma)=\sum_{i=0}^{2}(-1)^{i} \operatorname{Tr}\left(\sigma^{*} \mid H^{i}\left(X, \mathcal{O}_{X}\right)\right), \\
L(\sigma)=\sum_{i=1}^{M_{1}} a\left(P_{i}\right)+\sum_{j=M_{1}+1}^{M} a\left(P_{j}\right)+\sum_{k=1}^{N} b\left(C_{k}\right) .
\end{gathered}
$$

Here

$$
\begin{gathered}
a\left(P_{i}\right)=1 / \operatorname{det}\left(1-\sigma^{*} \mid T_{P_{i}}\right)=1 /\left(1-\zeta^{2}\right)\left(1-\zeta^{-1}\right), a\left(P_{j}\right)=1 /\left(1-\zeta^{3}\right)\left(1-\zeta^{-2}\right) \\
b\left(C_{k}\right)=\left(1-g\left(C_{k}\right)\right) /\left(1-\zeta^{-5}\right)-\left(\zeta^{-5} C_{k}^{2}\right) /\left(1-\zeta^{-5}\right)^{2}
\end{gathered}
$$

where $T_{P_{l}}$ is the tangent space to $X$ at $P_{l}, g\left(C_{k}\right)$ the genus of $C_{k}$ and $\zeta^{5}$ the eigenvalue of the action $\sigma_{*}$ on the normal bundle of $C_{k}$.

The first formula yields $L(\sigma)=1+\zeta^{-1}$ by the Serre duality $H^{2}\left(X, \mathcal{O}_{X}\right)$ $\cong H^{0}\left(X, \mathcal{O}\left(K_{X}\right)\right)^{\vee}$. Plugging this into the second formula for $L(\sigma)$, we get:

$$
1+\zeta^{-1}=M_{1} /\left(1-\zeta^{2}\right)\left(1-\zeta^{-1}\right)+M_{2} /\left(1-\zeta^{3}\right)\left(1-\zeta^{-2}\right)+N(1+\zeta) /(1-\zeta)^{2} .
$$

Multiplying this equality by denominators and simplifying it by the facts that $\zeta^{-1}=1-\zeta, \zeta^{3}=-1, \zeta^{2}=\zeta-1$, we obtain the following one which implies Lemma 1.6:

$$
3(1-\zeta)=\left(M_{1}+M_{2} / 2-3 N\right)(1-\zeta)
$$

Lemma 1.7. Let $X, \sigma, \zeta$ be as in Lemma 1.6. Assume that $\sum_{i=1}^{6} C_{i}$ is a linear chain of $\sigma$-stable smooth rational curves $C_{i}$ with $C_{i} \cdot C_{i+1}=1$. Set $P_{i}:=C_{i} \cap C_{i+1}$.

Then exactly one of $C_{i}$ is $\sigma$-fixed, say $C_{r}$, and the quintuplet $\sigma^{*} \mid P_{1}$, $\sigma^{*}\left|P_{2}, \cdots, \sigma^{*}\right| P_{5}$ of diagonalized local $\sigma^{*}$-actions, is equal to the unique portion 
of the following recursive sequence such that $\sigma^{*} \mid P_{r}=(1, \zeta)$ :

$$
\begin{gathered}
(\zeta, 1),(1, \zeta),\left(\zeta^{-1}, \zeta^{2}\right),\left(\zeta^{-2}, \zeta^{3}\right),\left(\zeta^{3}, \zeta^{-2}\right),\left(\zeta^{2}, \zeta^{-1}\right) \\
(\zeta, 1),(1, \zeta),\left(\zeta^{-1}, \zeta^{2}\right),\left(\zeta^{-2}, \zeta^{3}\right),\left(\zeta^{3}, \zeta^{-2}\right),\left(\zeta^{2}, \zeta^{-1}\right), \cdots
\end{gathered}
$$

Proof. Set $P_{i}:=C_{i} \cap C_{i+1}(1 \leq i \leq 5)$. If $C_{i}$ is $\sigma$-fixed, then $\sigma^{*} \mid P_{i}=(1, \zeta)$ with suitable local coordinates; otherwise $C_{i}$ contains exactly two $\sigma$-fixed points $P_{i-1}, P_{i}$ because $C_{i}$ is smooth rational and $\sigma$-stable, and $\sigma^{*} \mid P_{i}=\left(\zeta^{s}, \zeta^{1-s}\right)$ for some $s$ because $\sigma^{*} \omega=\zeta \omega$, where for $i=1,2, \cdots, 5$ (resp. for $i=0,1, \cdots, 4$ ), $\zeta^{s}$ (resp. $\zeta^{1-s}$ ) is the eigenvalue of the action $\sigma^{*}$ on the tangent to $C_{i}$ (resp. $C_{i+1}$ ) at $P_{i}$ and where $s, 1-s \neq 1(\bmod 6)$ because $C_{i}$ is not $\sigma$-fixed.

If $C_{i+1}$ is not $\sigma$-fixed, then $P_{i}$ and $P_{i+1}$ are the only two $\sigma$-fixed points on the smooth rational curve $C_{i+1}$ and hence $\sigma^{*} \mid P_{i+1}=\left(\zeta^{s-1}, \zeta^{2-s}\right)$. Now Lemma 1.7 is clear.

\section{§. Examples of Index 3 and Actual Type $\mathbf{A}_{17}$}

In the present section, we shall construct rational log Enriques surfaces of index 3 and actual Type $A_{17}$ (cf. Theorem 2).

Example 2.1 (index 3 and actual Type $\left.A_{17}\right)$. Let $\left(S_{3}, g_{3}, \Delta_{3}\right)$ be the Shioda-Inose's triplet in [OZ1, Example 1], where $S_{3}$ is the unique algebraic K3 surface of Picard number 20 and discriminant 3 (cf. [SI] or [V]), $g_{3}$ is an order-three automorphism on $S_{3}$ such that $g{ }_{3}^{*} \omega=\zeta_{3} \omega$ with a non-zero holomorphic 2-form $\omega$ and $\zeta_{3}=\exp (2 \pi \sqrt{-1} / 3)$, and $\Delta_{3}$ is a reduced simple normal crossing divisor on $S_{3}$ of Dynkin type $D_{19}$ as follows:

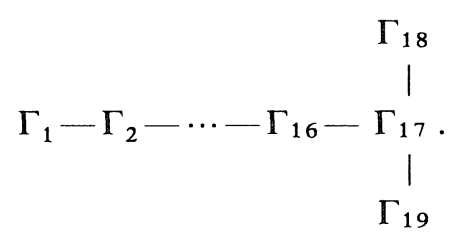

Note that $g_{3}$ acts trivially on $\operatorname{Pic} S_{3}$ such that the fixed locus (point wise)

$$
\left(S_{3}\right)^{g_{3}}=\operatorname{Supp}\left(\sum_{i=1}^{6} \Gamma_{3 i-1}\right) \amalg\left\{q_{0}, q_{1}, q_{3.4}, q_{6,7}, q_{9.10}, q_{12,13}, q_{15,16}, q_{18}, q_{19}\right\} .
$$

Here $q_{i . i+1}=\Gamma_{i} \cap \Gamma_{i+1}, q_{j} \in \Gamma_{j}$ and $q_{0}$ is a point not on $\Delta_{3}$. 
For $i=1$ (resp. 2, or 3), let $\gamma_{i}: S_{3} \rightarrow S_{\gamma_{t}}$ be the contraction of $\Delta_{3}-\left(\Gamma_{18}+\Gamma_{19}\right)$ (resp. $\Delta_{3}-\left(\Gamma_{1}+\Gamma_{18}\right)$, or $\left.\Delta_{3}-\left(\Gamma_{1}+\Gamma_{19}\right)\right)$. Then $S_{\gamma_{i}}$ is a $\mathrm{K} 3$ surface with a Du Val singular point of type $A_{17}$. The point $\gamma_{i}\left(q_{0}\right)$ and the only singular point on $S_{\gamma_{i}}$, together with two points $\gamma_{1}\left(q_{18}\right), \gamma_{1}\left(q_{19}\right)$ (resp. $\gamma_{2}\left(q_{1}\right)$ and $\gamma_{2}\left(q_{18}\right)$, or $\left.\gamma_{3}\left(q_{1}\right), \gamma_{3}\left(q_{19}\right)\right)$ are the only fixed points of the induced action of $g_{3}$ on $S_{\gamma_{i}}$. Clearly the quotient surface $S_{\gamma_{i}} /\left\langle g_{3}\right\rangle$ is a rational log Enriques surface of index 3 and actual Type $A_{17}$.

\section{§3. Extend $A_{17}$ to $A_{18}$ or $D_{18}$}

Let $T$ be a rational $\log$ Enriques surface of index 3 and Type $A_{17}$. We employ the notation at the beginning of $\S 1$ and in Lemma 1.1:

$$
\begin{aligned}
& \pi: Y \rightarrow T, f: S \rightarrow T, g: X \rightarrow Y, \Sigma=f^{-1}(\operatorname{Sing} T), \Gamma=g^{-1}(\operatorname{Sing} Y), \\
& \langle\sigma\rangle=\operatorname{Gal}(Y / T), \sigma^{*} \omega=\zeta_{3} \omega .
\end{aligned}
$$

We also denote by $\Gamma(1)=\Sigma_{i=1}^{17} \Gamma_{i}$ where $\Gamma_{i} \cdot \Gamma_{i+1}=1$, the unique connected component of $\Gamma$ of Dynkin type $A_{17}$.

Lemma 3.1. Let $T$ be a rational log Enriques surface of index 3 and Type $A_{17}$. Then we have:

(1) $T$ is of actual Type $A_{17}$, i.e., $\Gamma=\Gamma(1)$.

(2) After relabelling $\Gamma_{i}$ as $\Gamma_{18-i}$ if necessary, the $\sigma$-fixed locus (point wise) $X^{\sigma}$ is a disjoint union of six curves $\Gamma_{3 i-1}(1 \leq i \leq 6)$ and nine isolated points.

(3) The pair $(X, \sigma)$ is isomorphic to Shioda-Inose's pair $\left(S_{3}, g_{3}\right)$ defined in [OZ1, Example 1] (see also Example 2.1). In particular, $X$ is isomorphic to the unique K3 surface of Picard number 20 and discriminant 3 , and $\sigma$ acts trivially on Pic $X$.

Proof. Let $\Gamma(i)(1 \leq i \leq k)$ be all of connected components of $\Gamma$. Since $\rho(X) \leq 20$, one has $k \leq 3$ and each $\Gamma_{j}(j \geq 2)$ is of Dynkin type $A_{1}$ or $A_{2}$. By Lemma 1.2, each $\Gamma(i)$ is $\sigma$-stable. Since $3=\operatorname{ord}(\sigma)$ is coprime to the order of the graph-automorphism group $\mathbb{Z} / 2 \mathbb{Z}$ of a Dynkin diagram $A_{n}(n \geq 2)$, each irreducible component of $\Gamma$ is $\sigma$-stable. Hence each $\Gamma(i)$ contains at least one $\sigma$-fixed curve (cf. Lemmas 1.2 and 1.4).

By Lemmas 1.2 and 1.4, the set of $\sigma$-fixed curves contained in $\Gamma(1)$, is equal to $\sum_{i=1}^{6} \Gamma_{3 i-1}$ after relabelling if necessary. Since $X^{\sigma}$ can not contain more than six (smooth rational) curves [OZ1, Theorem 3, Example 1 and Remark 
3], one has $\Gamma=\Gamma(1)$. Now, (2) and (3) follow from [ibid.]. This proves Lemma 3.1 .

Remark 3.2. (1) Since $\sigma^{*} \mid \mathrm{Pic} X=\mathrm{id}$, each smooth rational curve, especially each component of $\Gamma_{i}$, is $\sigma$-stable. Hence one has the following (cf. Lemma 1.4(3)):

$$
X^{\sigma}=\operatorname{Supp}\left(\sum_{i=1}^{6} \Gamma_{3 i-1}\right) \amalg\left\{p_{1}, p_{3,4}, p_{6,7}, p_{9.10}, p_{12.13}, p_{15,16}, q_{1}, q_{2}, q_{3}\right\}
$$

where $p_{i, i+1}=\Gamma_{i} \cap \Gamma_{i+1}, p_{1} \in \Gamma_{1}$ and $q_{j}$ 's are points not on $\Gamma$.

(2) Since the discriminant of $X$ is 3 , for any 20 curves $C_{i}$ on $X$ one has $\operatorname{det}\left(C_{i} \cdot C_{j}\right)=-3 n^{2}$ for some non-negative integer $n$. Here $n$ is the index of the sublattice $\sum_{i=1}^{20} \mathbb{Z} C_{i}$ in the lattice $\operatorname{Pic} X$ when $C_{i}$ 's are linearly independent, and zero otherwise.

The rest of this section is devoted to the proof of the following:

Proposition 3.3. Let $T, S, \Sigma, Y, X, \Gamma$ be as in Lemma 3.1. Then there is a smooth rational curve $H$ on $X$ such that $H+\Gamma$ is of Dynkin type $A_{18}$ or $D_{18}$.

By Remark 3.2, $\Sigma$ consists of four connected components $\Sigma(1):=\Pi_{1}+\Sigma_{2}$ $+\Sigma_{5}+\Sigma_{8}+\Sigma_{11}+\Sigma_{14}+\Sigma_{17}, \Lambda_{i}(i=1,2,3)$ with the following dual graph:

$$
\Pi_{1}-\Sigma_{2}-\Sigma_{5}-\Sigma_{8}-\Sigma_{11}-\Sigma_{14}-\Sigma_{17}, \Lambda_{1}, \Lambda_{2}, \Lambda_{3} .
$$

Here $\Pi_{1}^{2}=-2, \Sigma_{2}^{2}=-3, \Sigma_{i}^{2}=-2(i=5,8,11,14), \Sigma_{17}^{2}=-4, \Lambda_{j}^{2}=-3(j=1,2,3)$.

The canonical cover $\pi: Y \rightarrow T$ induces a rational map $\pi: X \cdots \rightarrow S$ such that after relabelling if necessary, $\Gamma_{3 i-1}(1 \leq i \leq 6)$ on $X$ is the strict transform of $\Sigma_{3 i-1}$ and that $\Lambda_{j}$ is mapped to by $\pi^{-1}$, the three $\sigma$-fixed points $q_{j}$ which do not lie on $\Gamma$.

Lemma 3.4. Let $T, S, \Sigma$ be as in Lemma 3.1.

(1) One has $3\left(K_{S}+\Sigma^{*}\right) \sim 0$ where $\Sigma^{*}:=\left(\Pi_{1}+2 \Sigma_{i=1}^{6} \Sigma_{3 i-1}+\Sigma_{j=1}^{3} \Lambda_{j}\right) / 3$.

(2) $K_{S}^{2}=-3$ and $\rho(S)=13$.

(3) For any $(-1)$-curve $E$ on $X$ one has $E \cdot \Sigma^{*}=1$. If $H$ is an irreducible curve on $X$ with $H^{2}<0$, then $H$ is either a component of $\Gamma$ or a (-1)-curve.

Proof. (1) follows from the fact that $0 \sim f^{*}\left(3 K_{T}\right)=3\left(K_{S}+\Sigma^{*}\right)$ while (2) 
follows from (1). (1) and the genus formula imply the first half of (3) and that a curve $H$ with $H^{2}<0$ either satisfies (3), or is a $(-2)$-curve disjoint from $\Gamma$. The latter case is impossible because $\sigma^{*} \mid \operatorname{Pic} X=$ id by Lemma 3.1. This proves Lemma 3.4 .

Now our Proposition 3.3 will follow from the following Lemmas 3.5-3.9.

Lemma 3.5. Let $T, S, \Sigma$ be as in Lemma 3.1. Then there is a (-1)-curve $E$, or two disjoint (-1)-curves $E_{1}, E_{2}$, or three disjoint (-1)-curves $E_{1}, E_{2}, E_{3}$, on $S$ such that one of the following cases occurs (after relabelling if necessary):

Case(1). $\quad E \cdot \Lambda_{1}=E \cdot \Gamma_{3 r-1}=1$ for either one of $r=1,2, \cdots, 6$,

Case(2). $E \cdot \Lambda_{1}=E \cdot \Lambda_{2}=E \cdot \Pi_{1}=1$,

Case(3). $\quad E_{2} \cdot \Lambda_{j}=1(j=1,2,3)$, and $\left(E_{1} \cdot \Pi_{1}, E_{1} \cdot \Lambda_{1}\right)=(1,2)$ or $(2,1)$,

Case(4). $\quad\left(E_{j} \cdot \Pi_{1}, E_{j} \cdot \Lambda_{j}\right)=(1,2)$ or $(2,1)(j=1,2,3)$,

Case(5). each of $\left(E_{j} \cdot \Pi_{1}, E_{j} \cdot \Lambda_{j}\right)(j=1,2)$ and $\left(E_{3} \cdot \Lambda_{3}, E_{3} \cdot\left(\Lambda_{1}+\Lambda_{2}\right)\right)$ equals $(1,2)$ or $(2,1)$,

Case(6). each of $\left(E_{1} \cdot \Pi_{1}, E_{1} \cdot \Lambda_{1}\right),\left(E_{2} \cdot \Lambda_{1}, E_{2} \cdot \Lambda_{2}\right)$ and $\left(E_{3} \cdot \Lambda_{1}, E_{3} \cdot \Lambda_{3}\right)$ equals $(1,2)$ or $(2,1)$,

Case(7). each of $\left(E_{1} \cdot \Pi_{1}, E_{1} \cdot \Lambda_{1}\right),\left(E_{2} \cdot \Lambda_{1}, E_{2} \cdot \Lambda_{2}\right)$ and $\left(E_{3} \cdot \Lambda_{2}, E_{3} \cdot \Lambda_{3}\right)$ equals $(1,2)$ or $(2,1)$.

Proof. Let $v: S \rightarrow \Sigma_{m}$ be a smooth blowing-down of smooth rational curves to points on some Hirzebruch surface $F_{m}$ of degree $m$. Since $K_{F_{m}}+v_{*} \Sigma^{*} \equiv 0$ (Lemma 3.4), $v_{*} \Sigma$ contains at least one horizontal component and is hence connected.

$\operatorname{Claim}(1) . \quad \operatorname{Supp} v(\Gamma)=\operatorname{Supp} v_{*} \Gamma$, that is, no connected component of $\Sigma$ is contracted to a point not lying on $v_{*} \Sigma$.

Suppose to the contrary that a maximum union $\Sigma^{\prime}$ of connected components of $\Sigma$ is contracted to a point $p$ not lying on $v_{*} \Sigma$ so that $v\left(\Sigma^{\prime}\right) \cap v\left(\Sigma-\Sigma^{\prime}\right)=\phi$. Factorize $v=v_{3} \circ v_{2} \circ v_{1}$ so that $v_{1}\left(\Sigma^{\prime}\right)$ is a $(-1)$-curve and $v_{2}$ is the blowing down of $v_{1}\left(\Sigma^{\prime}\right)$. Then we have $0=v_{1}\left(\Sigma^{\prime}\right) \cdot v_{1 *}\left(K_{S}+\Sigma^{*}\right)=-1-\alpha<0$, where $\alpha$ is the coefficient in $\Sigma^{*}$ of the proper transform $v_{1}^{\prime}\left(v_{1}\left(\Sigma^{\prime}\right)\right)$. This is a contradiction. So Claim (1) is true.

Claim(1) and its preceding argument imply that $v(\Sigma)$ is connected. So 
$v^{-1} v(\Sigma)$ is also connected. We can write $v^{-1} v(\Sigma)=\Sigma+E_{-1}$ where $E_{-1}$ is a union of (-1)-curves (Lemma 3.4). Now Lemma 3.5 follows from Lemma 3.4(3) and the fact that $E_{-1}$ consists of disjoint (-1)-curves.

Lemma 3.6. Cases (4)-(7) in Lemma 3.5 are impossible.

Proof. Lemma 3.6 can be proved by dividing Cases (4)-(7) into subcases and applying Remark 3.2(2). We illustrate our method by considering the following subcase of Case (6): $E_{1} \cdot \Pi_{1}=E_{2} \cdot \Lambda_{2}=E_{3} \cdot \Lambda_{3}=1, E_{j} \cdot \Lambda_{1}=2(j=1,2,3)$.

Denote by $G_{17+j}$ the strict transform on $X$ of $E_{j}$. Then $G_{17+j}$ has self intersection 2 and, is either an elliptic curve with an ordinary node or a rational curve with a cusp of type $(2,5)$. Moreover, $G_{18} \cdot \Gamma_{1}=G_{18} \cdot \Gamma=1$, $G_{19} \cdot \Gamma=G_{20} \cdot \Gamma=0$ and $G_{i} \cdot G_{j}=4$ for $i, j=18,19,20 ; i \neq j$. Set $G_{i}=\Gamma_{18-i}(i=1$, $2, \cdots, 17)$. Using "Mathematica", we get $\operatorname{det}\left(G_{i} \cdot G_{j}\right)=-516=-3 \times 2^{2} \times 43$. This contradicts Remark 3.2(2). This way, one can prove Lemma 3.6.

Lemma 3.7. Suppose Case(1) in Lemma 3.5 occurs. Then Proposition 3.3 is true.

Proof. Let $E$ with $E \cdot \Lambda_{1}=E \cdot \Sigma_{3 r-1}=1$ be as in Case(1). Denote by $F$ the strict transform on $X$ of $E$. Then $F$ is a smooth rational curve such that $F \cdot \Gamma_{3 r-1}=F \cdot \Gamma=1$. If $r=1$ (resp. $r=6$ ), then $F+\Gamma$ is of Dynkin type $D_{18}$ (resp. $A_{18}$ ), whence Proposition 3.3 is true.

Therefore, we may assume that $r=2,3,4$ or 5 . Set $\eta_{0}:=4 \Gamma_{3 r-1}+3\left(\Gamma_{3 r-2}\right.$ $\left.+\Gamma_{3 r}\right)+2\left(\Gamma_{3 r-3}+\Gamma_{3 r+1}+F\right)+\Gamma_{3 r-4}+\Gamma_{3 r+2}$. Applying the Riemann-Roch theorem, there is an elliptic fibration $\psi: X \rightarrow \mathbf{P}^{1}$ with $\eta_{0}$ as a fiber.

Case(1.1) $r=2$. Let $\eta_{1}$ be the fiber containing $\Gamma_{10}+\Gamma_{11}+\cdots+\Gamma_{17}$. By Lemma 1.5 and the fact that $\Gamma_{9} \cdot \eta_{i}=1(i=0,1), \eta_{1}$ fits either type(2) with $n=9$ or type(3) with $n=11$ there. For type(3), we let $H$ be a tip component in $\eta_{1}$ which meets $\Gamma_{17}$ but not $\psi$ 's cross-section $\Gamma_{1}$. Then $H+\Gamma$ is of Dynkin type $A_{18}$.

For type(2), the cross-section $\Gamma_{1}$ meets $\eta_{1}$ at a point on the unique component $G$ of $\eta_{1}$ which is not contained in $\Gamma$. Thus the smooth rational curve $G$ contains three $\sigma$-fixed points $G \cap \Gamma_{1}, G \cap \Gamma_{10}, G \cap \Gamma_{17}$, and is hence $\sigma$-fixed (Lemma 1.4). This contradicts Remark 3.2(1).

Case(1.2) $r=3$. Let $\eta_{1}$ be the fiber containing $\Gamma_{13}+\Gamma_{14}+\cdots+\Gamma_{17}$. By Lemma 1.5 and the argument in Case(1.1) for type(2) there, $\eta_{1}$ fits type(3) in 
Lemma 1.5 and either $\eta_{1}=F_{2}+\Gamma_{3}+2\left(\Gamma_{2}+\Gamma_{1}+F+\Gamma_{17}+\Gamma_{16}+\Gamma_{15}+\Gamma_{14}\right)+\Gamma_{13}$ $+F_{14}$ where $F_{j} \cdot \Gamma_{j}=F \cdot \Gamma_{1}=F \cdot \Gamma_{17}=1$, or $\eta_{1}=F_{17}^{\prime}+F_{17}+2\left(\Gamma_{17}+\Gamma_{16}+\Gamma_{15}\right.$ $\left.+\Gamma_{14}\right)+\Gamma_{13}+F_{14}$ where $F_{j} \cdot \Gamma_{j}=F_{17}^{\prime} \cdot \Gamma_{17}=1$ and the cross-section $\Gamma_{4}$ does not meet $F_{17}$. In the first (resp. second) subcase $F_{2}+\Gamma$ (resp. $F_{17}+\Gamma$ ) is of Dynkin type $D_{18}$ (resp. $A_{18}$ ).

Case(1.3) $r=4$. Let $\eta_{1}$ be the fiber containing $\Gamma_{1}+\Gamma_{2}+\cdots+\Gamma_{6}$. By Lemma $1.5, \eta_{1}$ fits type(3) in Lemma 1.5 , and either $\eta_{1}=F_{5}+\Gamma_{6}+2\left(\Gamma_{5}+\Gamma_{4}+\cdots\right.$ $\left.+\Gamma_{1}+F+\Gamma_{17}\right)+\Gamma_{16}+F_{17}$ where $F_{j} \cdot \Gamma_{j}=F \cdot \Gamma_{1}=F \cdot \Gamma_{17}=1$, or $\eta_{1}=F_{2}+\Gamma_{1}$ $+2\left(\Gamma_{2}+\Gamma_{3}+\Gamma_{4}+\Gamma_{5}\right)+\Gamma_{6}+F_{5}$ where $F_{j} \cdot \Gamma_{j}=1$.

In the first subcase $F_{17}+\Gamma$ is of Dynkin type $A_{18}$. In the second subcase, if the cross-section $\Gamma_{15}$ does not meet $F_{2}$ (resp. $F_{5}$ ) then $F_{2}+\Gamma$ is of Dynkin type $D_{18}$ (resp. then we are reduced to Case(1.1) with $F$ replaced by $F_{5}$ ). Hence Proposition 3.3 is true by the arguments in Case(1.1).

Case(1.4) $r=5$. Let $\eta_{1}$ be the fiber containing $\Gamma_{1}+\Gamma_{2}+\cdots+\Gamma_{9}$. By Lemma $1.5, \eta_{1}$ fits type(3) with $n=11$ there. Let $H\left(\neq \Gamma_{1}\right)$ be the tip component of $\eta_{1}$ meeting $\Gamma_{2}$. Then $H+\Gamma$ is of Dynkin type $D_{18}$. This proves Lemma 3.7.

Lemma 3.8. Suppose Case(2) in Lemma 3.5 occurs. Then Proposition 3.3 is true.

Proof. Let $E$ be as in Case(2). Denote by $F$ the strict transform on $X$ of $E$. Then $F$ is a smooth elliptic curve with $F \cdot \Gamma_{1}=F \cdot \Gamma=1$. Let $\eta_{1}$ be the fiber of the elliptic fibration $\Phi_{|F|}: X \rightarrow \mathbb{P}^{1}$ containing $\Gamma_{2}+\Gamma_{3}+\cdots+\Gamma_{17} . \mathbb{B y}$ Lemma $1.5, \eta_{1}$ fits either type(3) with $n=20$, or type(2) with $n=18$. The first subcase is impossible for $n \leq 17$ (cf. Lemma 1.5). In the second subcase, if we let $H$ be one of two tip components in $\eta_{1}$ which meets $\Gamma_{17}$, then $H+\Gamma$ is of Dynkin type $A_{18}$. This proves Lemma 3.8 .

Lemma 3.9. Suppose Case(3) in Lemma 3.5 occurs. Then Proposition 3.3 is true.

Proof. Let $E_{1}, E_{2}$ be as in Case(3). Denote by $F_{j}$ the strict transform on $X$ of $E_{j}$. Then $F_{2}$ is a smooth elliptic curve, while $F_{1}$ is a curve of self intersection 2 such that $\left(F_{1} \cdot \Gamma_{1}, F_{1} \cdot F_{2}\right)=(1,2)$ or $(2,1)$. Applying Lemma 1.5 to the elliptic fibration $\psi:=\Phi_{\left|F_{2}\right|}: X \rightarrow \mathbb{P}^{1}$ we see that there is a smooth rational curve $F_{3}$ on $X$ such that $F_{3} \cdot \Gamma_{1}=F_{3} \cdot \Gamma_{17}=1$ and $\eta_{1}:=F_{3}+\Gamma$ is a fiber of $\psi$ of Kodaira type $I_{18}$. Since $F_{1} \cdot F_{2}=F_{1} \cdot \eta_{1}$, we see that $\left(F_{1} \cdot \Gamma_{1}, F_{1} \cdot F_{2}\right)=(1,2)$ and $\left(E_{1} \cdot \mathbb{\Pi}_{1}\right.$, 
$\left.E_{1} \cdot \Lambda_{1}\right)=(1,2)$

$F_{2} \sim \eta_{1}$ implies that $\xi_{0} \sim \xi_{1}$ where $\xi_{0}:=3 E_{2}+\sum_{i=1}^{3} \Lambda_{i}, \quad \xi_{1}:=3 E_{3}+2 \Pi_{1}$ $+\Sigma_{i=1}^{6} \Sigma_{3 i-1}$ are " $\pi$-direct images" of $F_{2}, \eta_{1}$ and where $E_{3}$ is the $\pi$-image of $F_{3}$. (We note that the six isolated $\sigma$-fixed points form the set of the indeterminant or fundamental points of the rational map $\pi: X \cdots \rightarrow S$.) Hence there is an elliptic fibration $\varphi: S \rightarrow \mathbb{P}^{1}$ with $\xi_{i}$ as fibers.

Claim(1). $\varphi$ is multiple fiber free.

Since the fibration $\psi$ on the $\mathrm{K} 3$ surface $X$ is multiple fiber free, it suffices to show that the inverse on $X$ of each fiber $\left(\neq \xi_{0}, \xi_{1}\right)$ of $\varphi$ splits into three distinct fibers of $\psi$.

We note that both $F_{2}$ and $\eta_{1}$ are $\sigma$-stable because $\sigma^{*} \mid \operatorname{Pic} X=\mathrm{id}$ and hence $\sigma^{*}$ permutes fibers of $\psi$ and induces an automorphism $\sigma$ on the base curve $\mathbb{P}^{1}$ of $\psi$. So it suffices to show that the action of $\sigma$ on $\mathbf{P}^{1}$ is non-trivial because then $\psi\left(F_{2}\right), \psi\left(\eta_{1}\right)$ are the only $\sigma$-fixed points on $\mathbb{P}^{1}$ and $\sigma$ acts freely on the set of all fibers of $\psi$ minus $F_{2}, \eta_{1}$.

If the action of $\sigma$ on $\mathbf{P}^{1}$ is trivial then $\pi_{*} \eta=3 \xi$ for a general fiber $\eta$ of $\psi$ where $\xi=\pi(\eta)$. So $3 \xi$ is linearly equivalent to the " $\pi$-direct image" $\xi_{i}(i=0$, 1). This is impossible because there are infinitely many such $3 \xi$ but the $\varphi$ can have at most one multiple fiber by noting that the Kodaira dimension of $S$ is $-\infty$ and applying the canonical divisor formula for elliptic surfaces. This proves Claim(1).

By Claim(1) and by the canonical divisor formula, one has $K_{S}+\xi_{i} \sim 0$ $(i=0,1)$. Let $E$ be a $(-1)$-curve on $S$. Then $E \cdot \xi_{i}=1$ and hence $E \cdot \Sigma_{3 i-1}=E \cdot \Lambda_{j}=1$ for some $1 \leq i \leq 6$ and $1 \leq j \leq 3$. So we are reduced to Case(1) in Lemma 3.5 after relabelling $\Lambda_{j}$ as $\Lambda_{1}$. Thus Proposition 3.3 is true by Lemma 3.7. This completes the proof of Lemma 3.9 and also that of Proposition 3.3.

\section{$\S 4$. Proofs of Theorems}

First, we prove Theorem 2. Let $T$ be a rational log Enriques surface of index 3 and Type $A_{17}$. We shall use the notations $T, S, X, \Gamma$ in Lemma 3.1. By Proposition 3.3, there is a smooth rational curve $H$ on $X$ such that $H+\Gamma$ is of Dynkin type $A_{18}$ or $D_{18}$. By Lemma 3.1(3) and [OZII, Theorems 3 and 4], there is a smooth rational curve $F$ on $X$ such that $(X,\langle\sigma\rangle, F+H+\Gamma\rangle$ is 
isomorphic to Shioda-Inose's triplet $\left(S_{3},\left\langle g_{3}\right\rangle, \Delta_{3}\right)$ in Example 2.1. Thus $(X$, $\langle\sigma\rangle, \Gamma)$ is isomorphic to $\left(S_{3},\left\langle g_{3}\right\rangle, \Delta_{3}-\left(\Gamma_{18}+\Gamma_{19}\right)\right),\left(S_{3},\left\langle g_{3}\right\rangle, \Delta_{3}-\left(\Gamma_{1}+\Gamma_{18}\right)\right)$ or $\left(S_{3},\left\langle g_{3}\right\rangle, \Delta_{3}-\left(\Gamma_{1}+\Gamma_{19}\right)\right)$. Now Theorem 2 follows.

Theorem 3 follows from the above arguments or Theorem 2 .

Next, we prove Theorem 4. Suppose the contrary that $T$ is a rational $\log$ Enriques surface of index $I$ and Type $D_{17}$. We use the same notations as at the beginning of $\S 1$. So $\Gamma$ contains a connected component $\Gamma(1)$ of Dynkin type $D_{17}$ as follows:

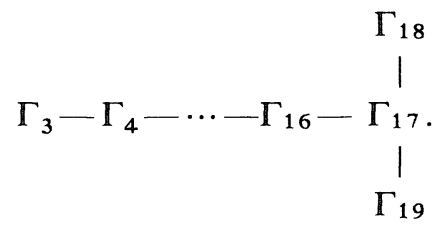

The existence of such $\Gamma(1)$ on $X$ implies that $\rho(X) \geq 18$. Thus Euler's Phi-function $\varphi(I) \leq \operatorname{rank} T_{X}=22-\rho(X) \leq 4$ (cf. "added in proof" at the end of [Z1]), and hence $I=2,4,8,12,3,6,5,10$. By Lemma 1.3 , it suffices to consider the cases $I=2,3,5$.

If $I=2$ then every singular point on the canonical cover $Y$ is of Dynkin type $A_{2 n-1}$ for some $n \geq 1$ (cf. [Z1, Lemma 3.1]). Hence $I \neq 2$. We can also use [OZ1, Lemma 3.2] to rule out the case $I=2$.

Consider the case $I=3$. Then each irreducible component $\Gamma_{i}$ in $\Gamma(1)$ is $\sigma$-stable because $3=\operatorname{ord}(\sigma)$ is coprime with the order of the graph-automorphism group $\mathbb{Z} / 2 \mathbb{Z}$ of $\Gamma(1)$ (cf. Lemma 1.2(2)). Now the intersection points of $\Gamma_{17}$ with $\Gamma_{16}, \Gamma_{18}$ and $\Gamma_{19}$ are $\sigma$-fixed. Hence the smooth rational curve $\Gamma_{17}$ is $\sigma$-fixed. Applying Lemma $1.4(2)$, we see that $\Gamma_{5}, \Gamma_{8}, \Gamma_{11}, \Gamma_{14}, \Gamma_{17}$ are the only $\sigma$-fixed curves in $\Gamma(1)$. Applying Lemma $1.4(3)$ to $C:=\Gamma_{3}$, we get a contradiction (cf. Lemma 1.2(3)). So the case $I=3$ is impossible.

Consider the case $I=5$. As in the case $I=3$, each irreducible component $\Gamma_{i}$ of $\Gamma(1)$ is $\sigma$-stable and $\Gamma_{17}$ is $\sigma$-fixed. Applying [OZ5, Lemma 1.6] which is an analogy of Lemma 1.4 for the case $I=5$, we see that $\Gamma_{7}, \Gamma_{12}, \Gamma_{17}$ are the only $\sigma$-fixed components in $\Gamma(1)$. This contradicts [OZ5, Lemmas 1.2 and 1.6] which are analogies of Lemmas 1.2 and 1.4, applied to the linear chain $\Gamma_{3}+\Gamma_{4}+\Gamma_{5}$. So $I=5$ is impossible. This completes the proof of Theorem 4. 
Finally, we prove Theorem 1. Suppose the contrary that $T$ is a rational $\log$ Enriques surface of index $6 p$ and Type $A_{17}$ for some $p \geq 1$. In view of Lemma 1.3, it suffices to consider the case $p=1$.

We shall employ the notation $\pi: Y \rightarrow T, \operatorname{Gal}(Y / T)=\langle\sigma\rangle, g: X \rightarrow Y$, $\Gamma=g^{-1}(\operatorname{Sing} Y)$ at the beginning of $\S 1$ and in Lemma 1.1. By Lemma 1.3, $T_{3}:=Y /\left\langle\sigma^{2}\right\rangle$ is a rational $\log$ Enriques surface of index 3 and Type $A_{17}$. In view of Lemma $3.1, T_{3}$ is of actual Type $A_{17}$, i.e., $\Gamma=\Gamma(1)=\sum_{i=1}^{17} \Gamma_{i}$ where $\Gamma_{i} \cdot \Gamma_{i+1}=1$. By Lemma 1.2, the fixed locus $X^{\sigma}$ is a subset of $\Gamma$.

Now applying Lemma 1.7 and using the fact that each $\sigma$-stable but not $\sigma$-fixed smooth rational curve has exactly two $\sigma$-fixed points, we see that $X^{\sigma}$ is equal to one of the following three sets, after relabelling $\Gamma_{i}$ as $\Gamma_{18-i}$ if necessary, where $p_{i, i+1}=\Gamma_{i} \cap \Gamma_{i+1}, p_{j} \in \Gamma_{j}$ :

$\operatorname{Supp}\left(\Gamma_{1}+\Gamma_{7}+\Gamma_{13}\right)$

$\amalg\left\{p_{2,3}, p_{3.4}, p_{4,5}, p_{5,6}, p_{8,9}, p_{9,10}, p_{10,11}, p_{11,12}, p_{14,15}, p_{15,16}, p_{16,17}, p_{17}\right\}$,

$\operatorname{Supp}\left(\Gamma_{2}+\Gamma_{8}+\Gamma_{14}\right)$

$\amalg\left\{p_{1}, p_{3.4}, p_{4,5}, p_{5,6}, p_{6.7}, p_{9,10}, p_{10,11}, p_{11,12}, p_{12.13}, p_{15,16}, p_{16,17}, p_{17}\right\}$,

$\operatorname{Supp}\left(\Gamma_{3}+\Gamma_{9}+\Gamma_{15}\right)$

U $\left\{p_{1}, p_{1,2}, p_{4,5}, p_{5,6}, p_{6.7}, p_{7,8}, p_{10.11}, p_{11,12}, p_{12,13}, p_{13,14}, p_{16,17}, p_{17}\right\}$.

By Lemma 1.7, in all these three cases, we have $M_{1}=M_{2}=6, N=3$ in the notations of Lemma 1.6. This contradicts the equality in Lemma 1.6. Therefore, Theorem 1 is true.

\section{References}

[A] Alexeev, V. A., Boundedness and $K^{2}$ for log surfaces, Intern. J. Math., 5 (1995), 779-810.

[AS1] Atiyah, M. F. and Segal, G. B., The index of elliptic operators: II, Ann. of Math., 87 (1968), 531-545.

[AS2] Atiyah, M. F. and Singer, I. M., The index of elliptic operators: III, Ann. of Math., 87 (1968), 546-604.

[B1] Blache, R., The structure of l.c. surfaces of Kodaira dimension zero, I, J. Alg. Geom., 4 (1995), 137-179.

[Br] Brieskorn, E., Rationale Singularitäten komplexer Flächen, Invent. Math., 4 (1968), 336-358.

[Ka] Kawamata, Y., Matsuda, K. and Matsuki, K., Introduction to the minimal model problem, Adv. Stud. in Pure Math., 10 (1987), 283-360.

[Ko] Kollár, J., Flips and abundance for algebraic threefolds, Astérisque, 211 (1992).

[O1] Oguiso, K., On algebraic fiber space structures on a Calabi-Yau 3-fold, Intern. J. Math., 4 (1993), 439-465.

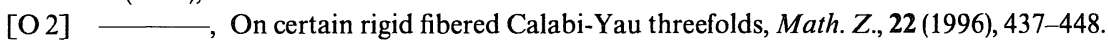


[O 3] Oguiso, K., A remark on the global indices of Q-Calabi-Yau 3-folds, Math. Proc. Camb. Phil. Soc., 114 (1993), 427-429.

[O 4] - On the complete classification of Calabi-Yau three-folds of Type $I I I_{0}$, in: Higher dimensional complex varieties, Proc. Intern. Conf. Trento 1994 (T. Peternell and M. Andreatta eds), 329-340.

[O 5] - Calabi-Yau threefolds of quasi-product type, Docum. Math., to appear.

[OZ1] Oguiso, K., and Zhang, D. -Q., On the most algebraic K3 surfaces and the most extremal log Enriques surfaces, Amer. J. Math., 118 (1996), 1277-1297.

[OZ2] - On extremal log Enriques surfaces, II, Tohoku Math. J., to appear.

[OZ3] - On the complete classification of extremal log Enriques surfaces, Math. Z., to appear.

[OZ4] - On Vorontsov's theorem on K3 surfaces with non-symplectic group actions, Preprint 1997.

[OZ5] - K3 surfaces with order five automorphisms, Preprint 1997.

[R] Reid, M., Campedelli versus Godeaux, in: Problems in the Theory of Surfaces and their Classification, Trento, October 1988, (F. Catanese, et al. eds.) Academic Press, 1991, pp. $309-365$.

[S] Shioda, T., On elliptic modular surfaces, J. Math. Soc. Japan, 24 (1972), 20-59.

[SI] Shioda, T. and Inose, H., On singular K3 surfaces, in: Complex analysis and algebraic geometry, Iwanami Shoten and Cambridge University Press (1977), pp. 119-136.

[V] Vinberg, E. B., The two most algebraic K3 surfaces, Math. Ann., 265 (1983), 1-21.

[Z1, Z2] Zhang, D. -Q., Logarithmic Enriques surfaces I; II, J. Math. Kyoto Univ., 31 (1991), 419-466; 33 (1993), 357-397.

[Z3] - Normal algebraic surfaces with trivial two or four times of the canonical divisor, Intern. J. Math., to appear. 